\title{
POLAROGRAPHIC METHODS OF ANALYSIS
}

\author{
WikToR Kemula \\ Department of Inorganic Chemistry, University of Warsaw, Warsaw, Poland
}

\section{INTRODUGTION}

The polarographic method invented by J. Heyrovský over 40 years ago has been associated with many successful achievements in many branches of physical and analytical chemistry, particularly in electrochemistry. Following the construction of the polarograph (with the excellent properties of Kučera's dropping mercury electrode incorporated) by Heyrovský and Shikata, recording the current-voltage curves has become much simplified contributing thereby to the rapid development of the polarographic method. To date over 20000 papers have been published in this discipline and further applications of the method continue to be investigated. The major practical application of polarographic technique is in analytical chemistry since it makes possible, in a simplified way, the detection and estimation of elements and compounds when present in very small concentrations.

\section{REGENT DEVELOPMENTS IN POLAROGRAPHIC METHODS}

The sensitivity of conventional polarography is such that measurements of concentration of different ions down to about $10^{-5} \mathrm{moles} / 1$. can be carried out. Determinations at greater dilutions are difficult, if not impossible, in consequence of the fact that the condenser current of the double layer of the dropping mercury electrode becomes great in comparison with the reduction current. This results in polarograms being obtained in which the step heights cannot be measured with sufficient accuracy.

In order to overcome this problem several techniques have been developed, notably the compensation method of Ilkovič and Semerano ${ }^{1}$ which permits determination of concentration between $10^{-5}$ and $10^{-6} \mathrm{moles} / \mathrm{l}$. and the "square wave" technique of Barker and Jenkins ${ }^{2}$ which extends the analytical range by a further order of magnitude. Both methods, however, suffer from certain disadvantages. The former method, among other things, requires provision for a rigorously constant drop-size while the latter necessitates the use of very complicated apparatus.

\section{Hanging mercury drop electrode methods}

An alternative approach to increase the sensitivity of analysis using the mercury electrode is to employ a stationary electrode and accumulate cations and anions on the electrode by pre-electrolysis. The idea is one of long standing but there have been few developments in this direction, mainly because it has proved extremely difficult to obtain reproducible results by such a method. The most obvious way to overcome the difficulties involved 


\section{WIKTOR KEMULA}

is by the use of a stationary (hanging) mercury drop, an arrangement which is particularly attractive since a spherical electrode is most amenable to theoretical treatment. However, a great difficulty has always been experienced in the use of this method owing to the penetration of the electrolytic solution between the mercury and the glass; a state of affairs which leads ultimately to the breaking up of electrical contact. This problem has been overcome as reported by us earlier ${ }^{3}$.

The accumulation on the mercury electrode by deposition of cations and anions by pre-electrolysis in stirred solution combined with subsequent stripping of formed amalgams or solid deposits on mercury in an unstirred solution has been made possible by the development of modern high sensitive recording polarographs. The results achieved on these polarographs, and also high reproducibility of drop-size and deposition conditions have enabled the determination of various ions present in an analytical solution in concentrations of the order of $10^{-10}$ moles/litre.

\section{Deposition and stripping}

The stripping method is a very powerful one especially for trace analysis. Of the various deposition and stripping methods the simplest one is that which applies a potential varying linearly with time and is called chronovoltammetry. The method is simple and sensitive, but the precision is not high and the accuracy of results obtained varies from 10 to 50 per cent. In this method concentrations are determined from the peaks of the anodic stripping currents of metals from the amalgams formed by pre-electrolysis. There are several reasons for these comparatively high errors. One is the difficulty in measuring the "background" current from which the peak current is measured. The "background" current is due mainly to the capacitative current of the discharging of the double layer of the hanging mercury electrode (which is relatively significant when determining concentrations of the order of $10^{-7}$ mole/l. or less ${ }^{4}$. This capacitative current caused by the differential capacity of the electrical double layer of the hanging mercury drop depends also on the rate of the potential change. The second difficulty arises when the stripping current of the more electro-negative metals that have no time to be oxidized before the potential reaches the oxidation potential of the next metal present in the mercury drop; this can influence the results. Further details on the results of investigations of these phenomena can be found in the literature ${ }^{6}$.

\section{Anodic stripping and cathodic pre-electrolysis}

Figure 1 shows a typical $I=f(U)$ curve obtained by anodic stripping of the metals accumulated in the hanging mercury drop by cathodic preelectrolysis. Curve 1 (dotted) represents schematically the discharging current of the double layer of the HMD electrode in $0 \cdot 1 \mathrm{~N} \mathrm{KNO}_{3}$ solution. Curve 2 (dotted) shows the anodic current of zinc that would flow in the absence of cadmium ions in the solution and, consequently, cadmium atoms in the amalgam. It follows from this example that the precise determination of anodic peak heights of cadmium and copper is possible only by applying certain corrections, while the determination of a very small quantity of lead is practically impossible. 


\section{POLAROGRAPHIC METHODS OF ANALYSIS}

I shall not discuss in any detail the methods and various techniques which have been developed as these have already been reviewed in detail ${ }^{5}$. I intend to limit my lecture to the results obtained in my laboratory with my coworkers on the previously described techniques and new ones developed recently.

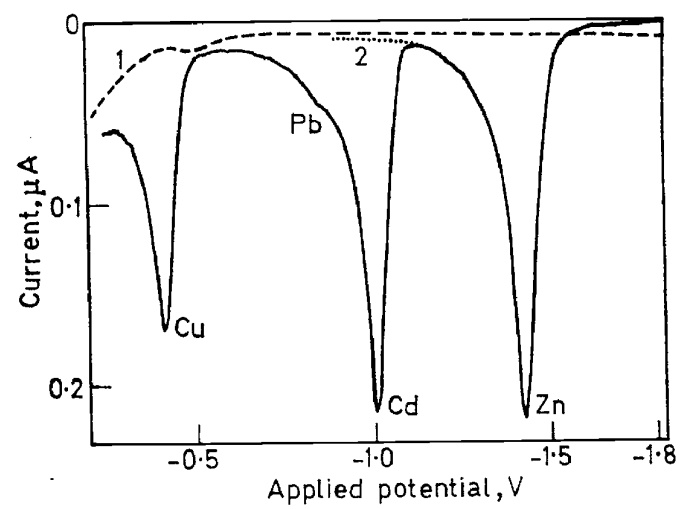

Figure 1. Anodic chronovoltammetric stripping after $5 \mathrm{~min}$ long pre-electrolytic deposition by $-1.8 \mathrm{~V}$. Background electrolyte $0.1 \mathrm{~N} \mathrm{KNO}$ containing $8 \times 10^{-8} \mathrm{~mole} / \mathrm{l}$. $\mathrm{Cu}^{2+}, 3 \times 10^{-9}$ mole $/ 1 . \mathrm{Pb}^{2+}, 8 \times 10^{-8}$ mole/l. $\mathrm{Cd}^{2+}$, and $8 \cdot 5 \times 10^{-8} \mathrm{~mole} / 1$. $\mathrm{Zn}$.

I would like to point out that the hanging mercury drop electrode (HMDE) technique has proved successful in performing analyses of various ions present in traces in waters ${ }^{7}$, salts ${ }^{3}$, metals ${ }^{3}$, acids ${ }^{8}$, and alkalies ${ }^{9}$ when cathodic pre-electrolysis and anodic stripping was done.

\section{Cathodic stripping and anodic pre-electrolysis}

Anodic pre-electrolysis and subsequent cathodic stripping was also successful in the determination of traces of chloride, bromide and iodide in waters, and chloride in concentrated acids, etc. ${ }^{10}$ An indirect method of evaluation of traces of mercury has been described as well. This method is based on diminution of the stripping peak of iodide in the presence of mercury ${ }^{11}$. Several organic compounds present in trace amounts in a solution on electrolysis also form insoluble deposits on mercury electrode and can be accumulated and subsequently stripped ${ }^{12}$.

The equations describing the dependence of stripping peak height on concentration of the investigated ion, on time of deposition, on stripping rate etc., have been calculated. The reproducibility of the results depends not only on the constancy of mercury drop-size, temperature, rate of stirring, and concentration of the background electrolyte ${ }^{3}$, but also on the quantity of the air present in the solution, surfactants, concentration of the more positive species of ions, etc. ${ }^{13}$

\section{Influence of double layer capacity}

The influence of the double layer capacity of the mercury electrode and the stripping rate of the metals deposited on the results of analysis remained still a problem, the importance of which grows, when the quantity of trace 
amount of the analysed species diminishes. Different workers have tried to solve this problem in different ways.

Usually, when stripping rate is low, the double layer capacity plays a smaller role, but at the same time peaks of metals stripped are also lower, although the stripping is more complete. By raising the stripping rate or when the size of the mercury drop is greater there is a possibility that not the whole quantity of the metal is stripped from the electrode (cf. Figure 1). This fact led different workers to the use of the solid metal or graphite electrodes covered with a thin layer of mercury ${ }^{14}$.

A major difficulty in stripping analysis of metals is the formation of intermetallic compounds of deposited metals with mercury or with other deposited metals ${ }^{3}$. To avoid these difficulties it is necessary to deposit only small amounts of metals in mercury. From our experience and from literature it follows that the simplest method of stripping analysis is to use pre-electrolysis and stripping with hanging mercury drop electrode.

\section{The "sluice" method"}

An improved apparatus described not long ago enables one to perform the analyses faster and more precisely by standard addition methods using the "sluice". This device makes it possible to add exact amounts of standard solution without the need to de-aerate the solution repeatedly ${ }^{15}$.

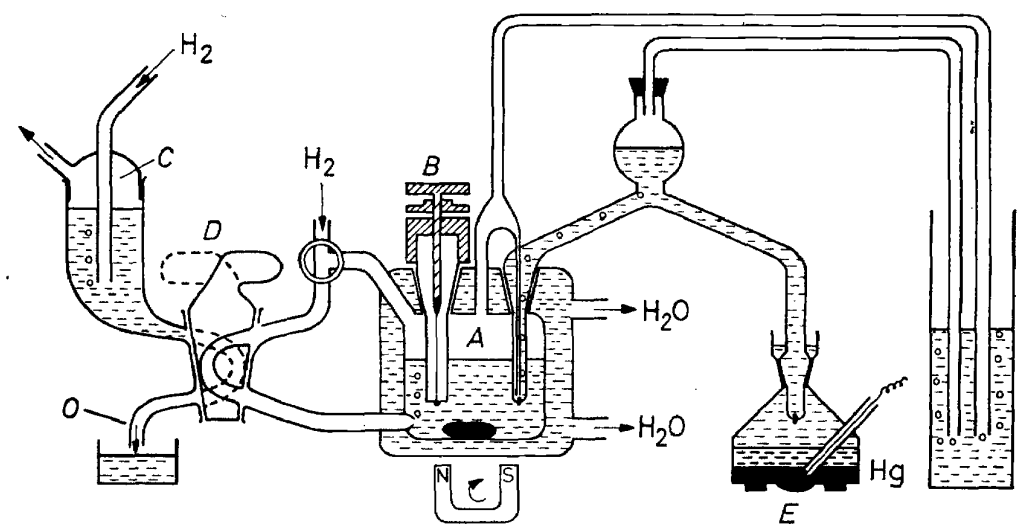

Figure 2. New set of apparatus for stripping analysis with pre-electrolysis and hanging mercury drop electrode. $A$, Electrolytic cell with magnetic stirrer; $B$, HMD electrode; $C$, Container of standard solution; $D$, "Sluice"; $E$, Reference electrode; $O$, Outlet.

The thorough de-aeration of solutions is very important, because the presence of oxygen in solutions causes uncontrolled diminution of stripping peaks owing to the reaction of amalgam formed with dissolved oxygen according to the equation

$$
\mathrm{Me}{ }^{\mathrm{II}}(\mathrm{Hg})+\mathrm{O}+\mathrm{H}_{2} \mathrm{O} \rightarrow(\mathrm{Hg})+\mathrm{Me}^{2+}+2 \mathrm{OH}^{-}
$$




\section{POLAROGRAPHIG METHODS OF ANALYSIS}

Similarly, mercury ions present in the solution reduce the concentration of ions deposited reacting with the metals in the amalgam according to equation

$$
\mathrm{Me}^{\mathrm{Ir}}(\mathrm{Hg})+\mathrm{Hg}^{2+} \rightarrow(\mathrm{Hg})+\mathrm{Me}^{2+}
$$

For this reason oxygen should be removed, and the use of an external reference electrode is advised. The HMD electrode should be dipped into the solution after de-aeration of the solution to avoid the dissolution of the mercury. The other more positive ions present in the solution can react in the same manner as mercury ions with the less electro-positive metals deposited in the HMD electrode. For example, if zinc is deposited at the mercury electrode, and the solution contains cupric ions, the following reaction can take place

$$
\mathrm{Zn}(\mathrm{Hg})+\mathrm{Cu}^{2+} \rightarrow \mathrm{Cu}(\mathrm{Hg})+\mathrm{Zn}^{2+}
$$

Consequently the quantitative result of the stripping is dependent on the time of interaction with reacting species dissolved and its concentration. In the last case the quantity of zinc in amalgam to be stripped is smaller, and that of copper increases, owing to the uncontrolled substitution.

The "sluice" method ${ }^{15}$ renders a precise determination of minute amounts of impurities possible without the prior ascertaining of the calibration curve for a given analysis. The method consists in introducing into the solution known quantities of single species dissolved in the same solution of background electrolyte as present in the solution to be analysed without renewed de-aeration, followed by dilution of the sample. Figure 3 shows an example of the quantitative evaluation of lead in pure potassium nitrate by this method ${ }^{15}$.

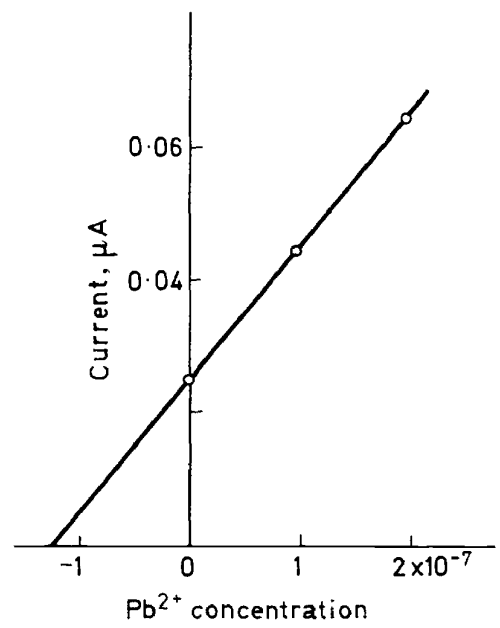

Figure 3. Determination of lead in pure potassium nitrate by "sluice" increment method". The quantity of lead present in solution is $1.3 \times 10^{-7} \mathrm{~Pb}^{2+}$. 
New stripping method

A closer look at the processes that are taking place at the HMDE leads to the conclusion that instead of recording the instantaneous maximal anodic current, more accurate results can be obtained by measuring the total charge that flows during the anodic stripping. The measurement of the charge corresponding to the stripping of some quantity of metal deposited initially at the HMDE is rather difficult because the quantities of electricity flowing in such processes are in the range of $1 \mu \mathrm{G}$, and even lower, for concentrations lower than $10^{-7}$ mole/l. The baseline with respect to which the integration has to be made is not a straight line and the stripping time of the metal from the HMDE is rather short, and usually ranges from several seconds to $1 \mathrm{~min}$.

From these conditions it follows that a high quality apparatus has to be used for charge measurement. Its sensitivity must be high enough to enable the measurement of a hundredth part of $1 \mu \mathrm{C}$ for full-scale deflection and its time constant should be in the range of $10^{-4} \mathrm{sec}$. This is the reason why instead of charge measurements we have applied a method of recording the potentials of the galvanic element formed from the HMDE with deposited metals and reference calomel or other electrode, when stripping process of deposited metals occurred.

If no current is consumed and the solution contains no oxygen or more electro-positive metal ions, and the amalgam does not react with the solvent, the stripping process will not occur. If, under such conditions, after metal deposition on the HMDE, this electrode is connected to the reference electrode through a large resistor (more than $10^{7} \Omega$ so that the stripping process is comparatively slow) the galvanic cell formed will have a voltage which can be measured with an electrometer (cf. Figure 4). Using such

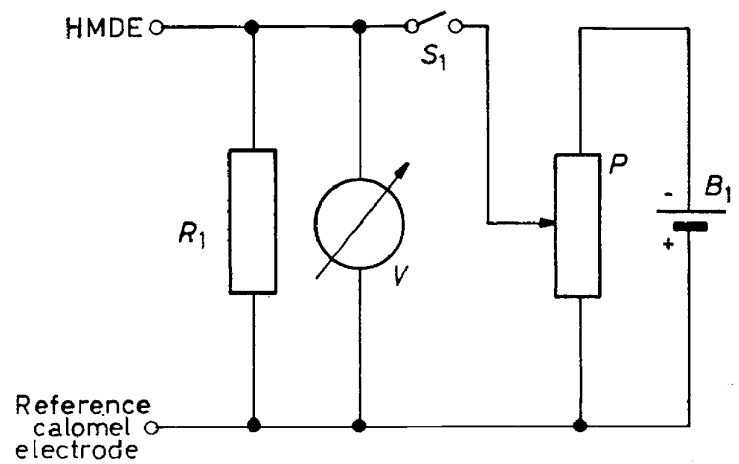

Figure 4. Flectrical circuit for $R=$ constant stripping method. HMDE hanging mercury drop electrode; $R_{1}$ stripping resistor $\left(10^{8}-10^{9} \Omega\right) ; V$ electrostatic dynamic voltmeter with an input resistance $10^{15 \Omega ; P}$ potentiometer for pre-electrolysis and stripping; $B_{1}$ battery; $S_{1}$ deposition current switch.

an arrangement, the initial stripping potential observed will be that of the most electro-negative metal. This lasts as long as this metal is present in the electrode. Then the potential drops to the stripping potential of the next more electro-negative metal. When this metal is almost completely 


\section{POLAROGRAPHIC METHODS OF ANALYSIS}

oxidized the potential drops to the stripping potential of the next more electro-positive metal and this is repeated until all the metals initially deposited are oxidized from the HMD electrode. The current intensity observed in the circuit of the applied constant resistor is proportional to the instantaneous voltage of the cell. Thus, the dependence of the voltage on the time, $U=f(t)$, is recorded, and the areas under each step correspond to the charges that flow during anodic stripping of the metals. The concentration measurements are simplified to the measurement of stripping times which are proportional to the length of the recorded step. This kind of measurement should be more precise than the peak-height measurement in the chronovoltammetric method as it is in reality with lead, present in very small quantities (cf. Figures 5 and 7). Diffusion, and some incidental

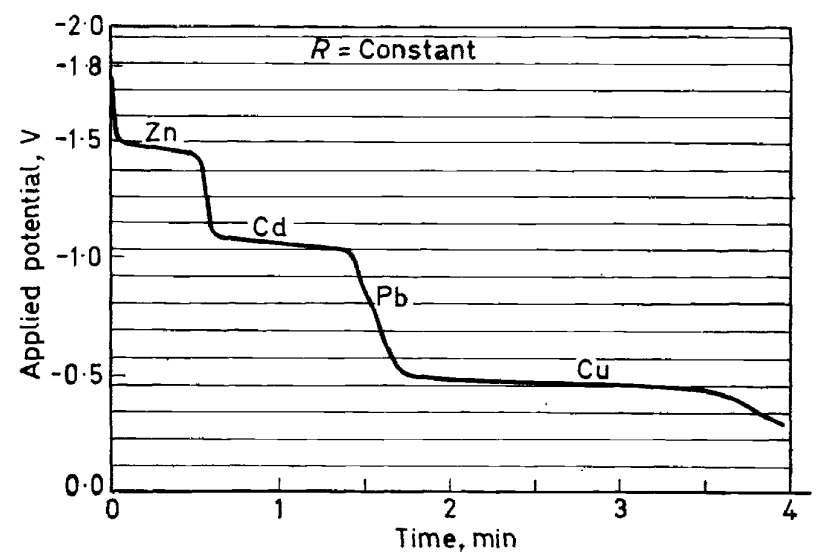

Figure 5. Anodic stripping curve obtained by $R=$ constant method. Time of pre-electrolysis and composition of the solution same as in Figure 1.

resistance which in voltammetry with linearly changing potential can change the peak-height, have no effect on the stripping time.

During the stripping time, the potential of the indicator electrode, in our case HMDE, is given by the Nernst equation, so that stripping should be selective and simultaneous dissolution of different metals should not occur as in chronovoltammetry. The rate of the double-layer discharge is dependent on the resistance of the circuit and the potential. These parameters affect the steepness of potential changes on the second curve. Since the potentials at the beginning and the end of the horizontal part of each step are similar, the capacitative current is also similar.

Determination of stripping time. To determine the concentrations of different ions on the basis of the recorded diagram, both the stripping times of metals and their voltages have to be taken into account. To some extent this complicates the comparison and evaluation of concentrations of different ions.

This method, which is characterized by constant resistance, can be modified in such a manner that the stripping current is the same for the stripping 


\section{WIKTOR KEMULA}

of all metals deposited in the HMDE. The concentrations of these ions will then be proportional only to their stripping times from the amalgam. This kind of stripping can be easily arranged if one inserts in the circuit (cf. Figure 6) a current source with the voltage exceeding by two orders of magnitude the stripping voltage of the most negative metal and simultaneously increase in the same ratio (two orders of magnitude) the resistance

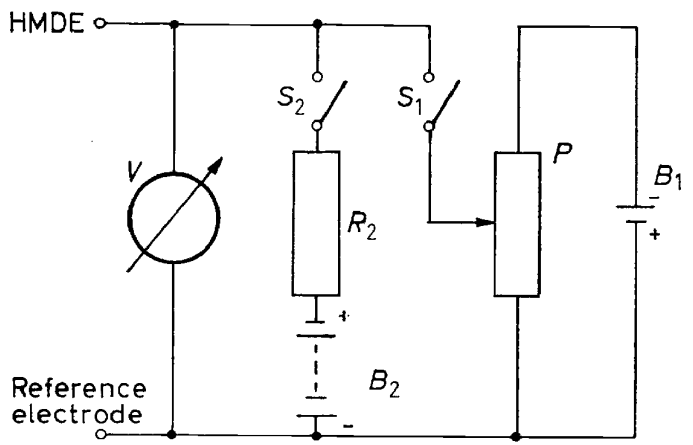

Figure 6. Electrical circuit for $I=$ constant stripping method. $R_{2}$ resistor fixing stripping current; $B_{2} 50 \div 200$ volt battery for stripping current; $S_{1}$ deposition switch: $S_{2}$ stripping current switch.

of the circuit. Such an arrangement enables the anodic stripping to be carried out at constant current. The stripping results obtained by this $(I=$ constant $)$ method are shown in Figure 7.

The experiments carried out by us confirm the possibility that well-defined steps can be worked out such that the determination of the stripping time becomes easy and precise. The superiority of these methods becomes evident if one compares the lead stripping step on these curves with that obtained by the chronovoltammetry method shown in Figure 1.

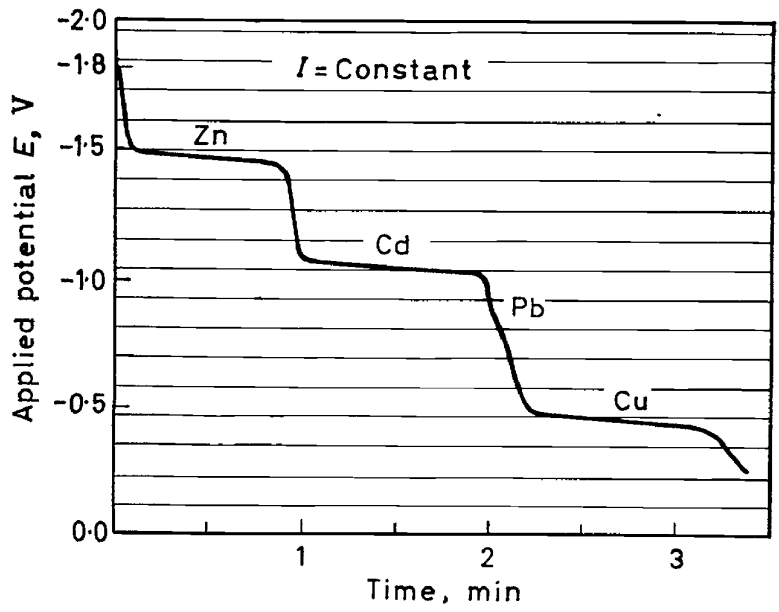

Figure 7. Anodic stripping curve obtained by $I=$ constant method. Pre-electrolysis time and solution composition as in Figure 1. 


\section{POLAROGRAPHIG METHODS OF ANALYSIS}

The measurement of the stripping time was graphical and analogous to that used in polarography. The steep part of the curve, more or less parallel to the time axis in the middle of the curve, was taken as the stripping time.

Another example is that in which small amounts of lead in cadmium can be estimated by plotting the corresponding $(a, b, c$ and $d)$ stripping curves of the solutions obtained by the chronovoltammetric and $I=$ constant methods (Figures 8 and 9). The difficulty arising here is in the measurement of precisely small concentrations of more positive ions in the presence of large quantities of more negative ones.

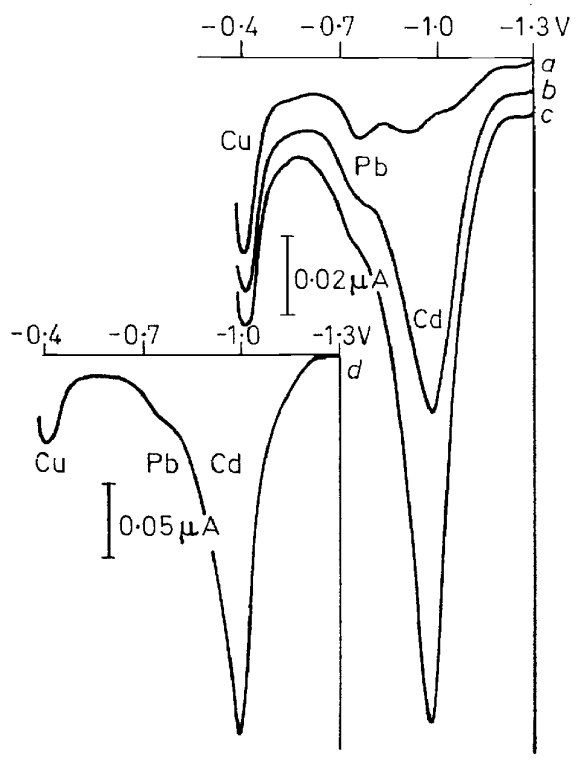

Figure 8. Determination of small amounts of lead in cadmium. Chronovoltammetric stripping curves of solutions containing: (a) $2 \times 10^{-8} ;(b) 1.2 \times 10^{-7} ;$ (c) $2.2 \times 10^{-7}$; and (d) $3.2 \times 10^{-7} \mathrm{~mole} / \mathrm{l}$. of cadmium in the $0.1 \mathrm{~N} \mathrm{KNO}_{3}$ background electrolyte containing $1.2 \times 10^{-7} \mathrm{Cu}^{2+}$ and $6 \times 10^{-8} \mathrm{~Pb}^{2+}$ mole/l.

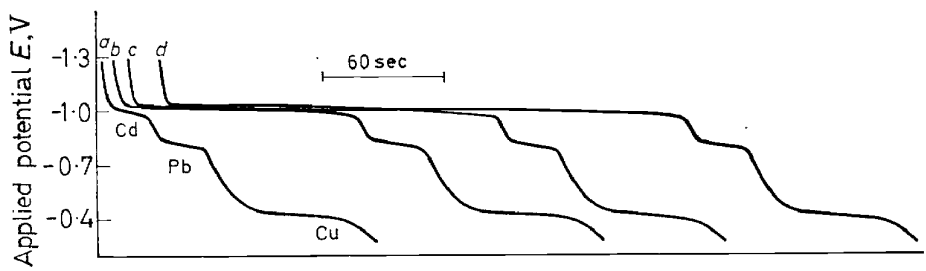

Figure 9. Determinations of small amounts of lead in cadmium using $I=$ constant method. Curves $a, b, c, d$ have the same significance and values as in Figure 8.

From comparison of the results it follows clearly that what was practically impossible to solve by chronovoltammetric stripping can be done by $I=$ constant method.

The reproducibility of numerous experimental curves was good and the error did not exceed 2 per cent. The accuracy of the stripping time deter- 
mination varied from 1 to 5 per cent depending on the length of step. The precision of the concentration determination by these methods was $2-10$ per cent. To obtain this accuracy, no oxygen or other substituting species had to be present in solution ${ }^{15}$. If such ions are present to diminish the effect of such reactions, the ratio of the flux of dissolved ions to the flux of deposited ions should be as high as possible (aboui 100). This means that the deposition of metal should be carried out rather intensively in vigorously stirred solutions and the oxidation current should be comparatively high. The effect of ions present in the solution that can interact with the metals of the amalgam is decreased by carrying out the oxidation process in quiet solution.

The recorded process of metal stripping permits the calculation of the quantity of electricity that flows in the time of metal stripping from the amalgam into the solution. This charge can be calculated from the curves obtained by the three methods mentioned (Figures 1, 5 and 7). From the data obtained it follows that the agreement of results is better in methods $R=$ constant and $I=$ constant; the results obtained by the chronovoltammetric methods were lower. It can be assumed that at the given scan rate $(0.8 \mathrm{~V} / \mathrm{min})$ only part of each metal was dissolved from the amalgam.

From these results it follows that especially recommended is the $I=$ constant method, where the stripping times are proportional to the concentrations of deposited metals and, consequently, to the concentrations of these metal ions in the solution.

The results obtained by new stripping methods are better, but still there are factors which influence these results, such as composition of dissolved analysed sample, diffusion of accumulated metals in the capillary, size of the drop retarding the complete stripping of deposited metals, continuing deposition of more positive ions during the stripping of more negative ones, etc.

The effect of stirring the solution during stripping was investigated next ${ }^{16}$. As was to be expected stirring the solution during deposition accelerated dissolution of metals from the amalgam, because HMD electrode was continuously shaken and ions formed as stripping products were immediately removed into the solution.

From Figure 10 it follows that stirring is favourable because the substitution time (although more effective) diminishes, but stirring has the disadvantage that it simultaneously raises the deposition rate of more positive ions. This causes distortion of the stripping curve in comparison to the curve obtained in quiet solution (Figure 10).

As was to be expected the peaks stripped in stirred solution were considerably higher and this could be confirmed experimentally (cf. Figure 10). From this figure it follows also that stripping from small drop (mercury surface $=0.65 \mathrm{~mm}^{2}$ ) even in unstirred solution shows good peak resolution (curve 1). Stripping with stirring does not further improve the resolution of peaks (curve 2). When HMD electrode is larger and has twice as big surface $\left(1.29 \mathrm{~mm}^{2}\right)$ stripping in quiet solution (curve 3 ) is visibly retarded, but improves in the stirred solution (curve 4). It can be seen also that peaks in stirred solutions are higher than in quiet ones. Besides difficulties with the satisfactory resolution of peaks, there are others too, especially in very dilute solutions. 


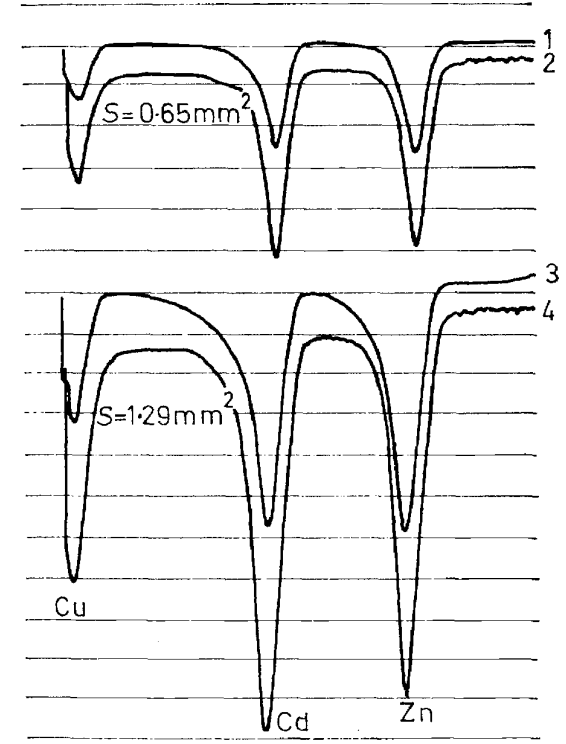

Figure 10. Anodic stripping curves in quiet and stirred solutions. Curves 1 and 3 refer to the unstirred and curves 2 and $\mathbf{4}$ to the stirred solutions.

\section{DIFFERENTIAL METHODS}

In the method discussed above the problem of quantitative analysis is based on the measurements of the peak heights and is complicated especially when very low concentrations have to be analysed by the necessity of exact knowledge of the double layer capacity of the mercury electrode, which is not constant and influences the zero-line of the measured peak.

There are different methods of measuring the peak heights caused by the change of the base line. We have recently reported a simple one ${ }^{17}$. All the difficulties mentioned so far besides other ones like the effect of the presence of surfactants in solutions ${ }^{18}$, etc. led us to investigate the possibility of applying in our case a differential (subtractive) method, enabling us to eliminate generally these noxious phenomena.

In conventional polarography and recently in oscillopolarography an application of the differential method has been described ${ }^{19}$ which enables us to subtract the results obtained in exactly the same conditions from two electrodes dipped in two solutions: one is solution of background electrolyte and the other is the analysed sample dissolved in the same background electrolyte.

Such apparatus, owing to the necessity to de-aerate carefully the solutions and to avoid the presence of different other impurities like traces of surfactants in both cells should be very complicated. To simplify the procedure ${ }^{20}$ we have decided to choose another way - to dip two electrodes of exactly the same size and properties into the same electrolytic cell, but deposit different quantities of metals on both electrodes, switching on the electrodes for different time in the circuit. 


\section{WIKTOR KEMULA}

The subsequent subtractive stripping enables one to record only the differences in quantities of metals accumulated by pre-electrolysis, but excludes the influence of the eventual presence of impurities, which are not differently accumulated during pre-electrolysis.

This simple idea could be realized by the circuit given in the Figure 11. High input resistance galvanometer $G_{1}$ measures the difference if the currents are flowing in two circuits during the stripping. The recording galvanometer $G_{2}$ measures the sum of both stripping currents from the two electrodes. The results obtained are given in Figures 12 and 13.

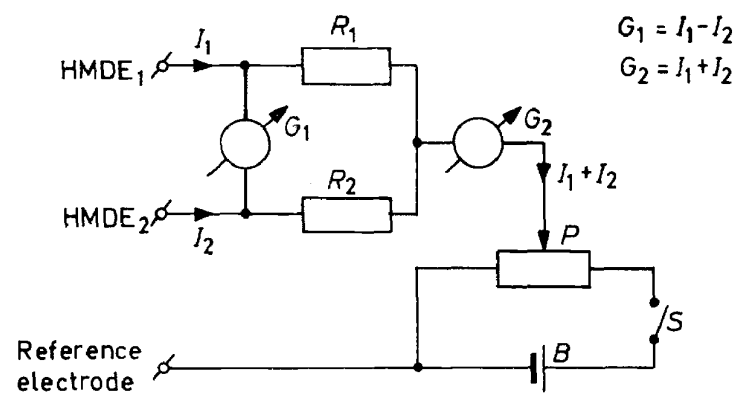

Figure 11. The circuit for differential (subtracting) recording of metals deposited by preelectrolysis on two separate HMD electrodes. $R_{1}, R_{2}$ resistors; $G_{1}$ high input resistance galvanometer; $G_{2}$ high sensitivity recording galvanometer; $P$ potentiometer; $B$ battery; $S$ switch

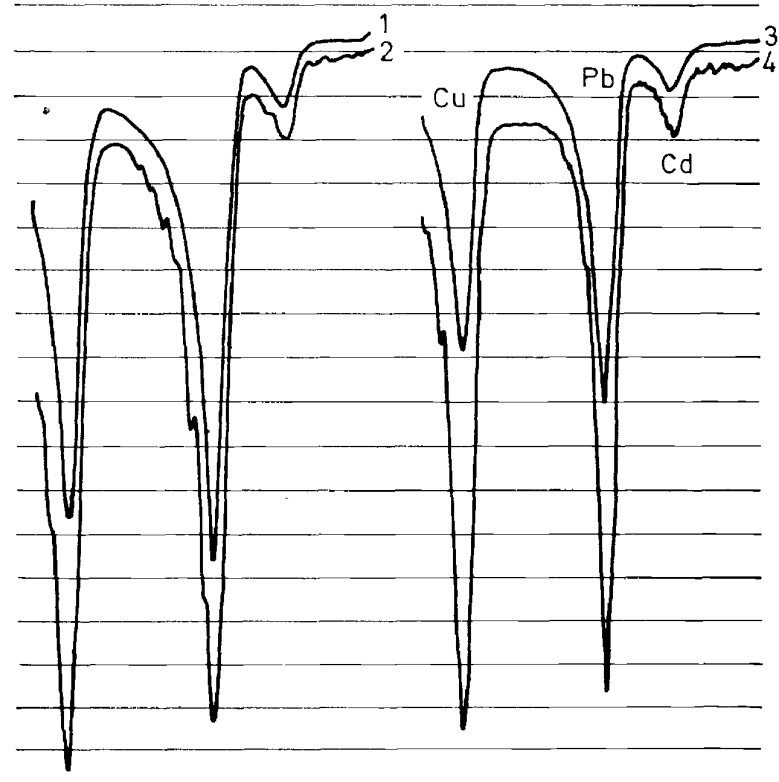

Figture 12. The comparison of the $I_{1}-I_{2}$ and $I_{1}+I_{2}$ curves. The solution contained 10-6 $\mathrm{Gu}^{2+}, 10^{-6} \mathrm{~Pb}^{2+}$ and $10^{-7} \mathrm{Cd}^{2+}$ in $0.1 \mathrm{~N} \mathrm{KNO}_{3}+10^{-4} \mathrm{~N} \mathrm{HNO}_{3}$. Stripping rate in $I_{1}+I_{2}$ curves: curves 1 and $2,0.8$ volt $/ \mathrm{min}$; curves 3 and $4,0 \cdot 4$ volt $/ \mathrm{min}$. 


\section{POLAROGRAPHIG METHODS OF ANALYSIS}

This set of apparatus enabled us to check the dissolution rate and peak heights, depending on the rate of potential change, the influence of stirring on the peak heights, etc.

From Figure 13 it follows that this arrangement makes it possible also to compensate completely for the double layer capacity and all impurities like traces of oxygen, surfactants, etc.

The high repeatability of stripping results permits one to suppose that on application of differential integrator according to the scheme in Figure 14 digital results can be obtained without the need for recording.

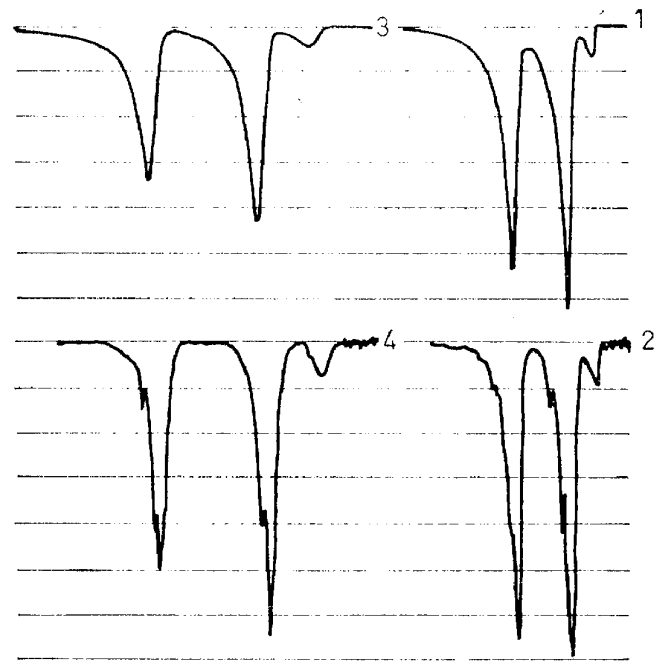

Figure 13. $I_{1}-I_{2}$ curves recorded simultaneously with $I_{1}+I_{2}$ curves in Figure 12 .

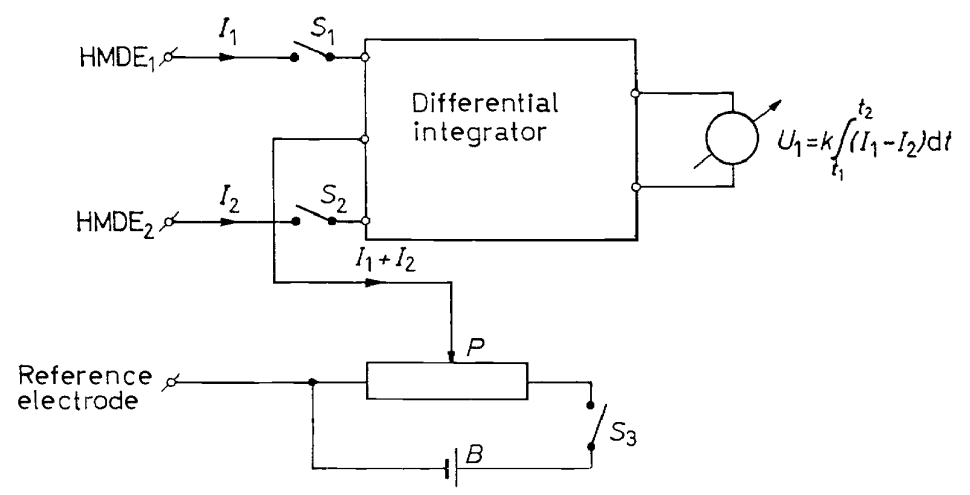

Figure 14. Electrical circuit for differential integrator set.

The investigations undertaken are promising and an apparatus has been developed (Figure 14). The important condition is to keep long lasting stability of factors, influencing deposition and stripping. We hope to be able to publish these results in the near future. 


\section{WIKTOR KEMULA}

\section{References}

1 D. Ilkovič and G. Semerano. Coll. Czech. Chem. Comm., 4, 176 (1932).

2 G. G. Barker and I. L. Jenkins. Analyst. 77, 685 (1952).

3 W. Kemula. Advances in Polarography, Vol. 1, p. 105. Pergamon Press, Oxford, 1960.

W. Kemula and Z. Kublik. Advances in Analytical Chemistry and Instrumentation, Vol. 2, Interscience, New York, 1963.

4 W. Kemula and J. W. Strojek. Chem. Anal. Warsaw 10, 1327 (1965).

5 Charles N. Reilley and others in Treatise on Analytical Chemistry I. M. Kolthoff and Philip

I. Elving (Eds.), Part 1, Volume 4, pages 2075-2671. Interscience, New York, 1963.

L. Meites. Polarographic Techniques 2nd Edition. John Wiley and Sons, 1965.

6 W. Kemula and J. W. Strojek. Chem. Anal. Warsaw 10, 1327 (1965); J. Electroanalyt. Chem. 12, 1 (1966).

7 Z. Kublik. Acta Chim. Hung. 27, 79 (1961).

G. Macchi. J. Electroanalyt. Chem. 9, 290 (1965).

8 W. Kemula and S. Gtodowski. Chem. Anal. Warsaw 8, 369 (1963).

H. Specker, G. Schiewe and H. Trüb. Z, Anal. chem. 190, 144 (1962).

9 W. Kemula and S. Gtodowski. Chemia Anal. 11, 403 (1966).

10 W. Kemula, Z. Kublik, and J. Taraszewska. J. Electroanalyt. Chem. 6, 119 (1963).

11 H. Berge and S. Głodowski. Chem. Anal. Warsaw 8, 361 (1963).

12 W. Kemula, Z. Kublik and A. Axt. Roczniki Chem., 35, 1009 (1961).

13 W. Kemula and J. W. Strojek. Chem. Anal. Warsaw 10, 1327 (1965);

J. Electroanalyt. Chem. 12, 1 (1966).

14 R. N. Adams in Treatise on Analytical Chemistry I. M. Kolthoff and P. I. Elving (Eds.) Part 1, Vol. 4, pages 2381-2416. Interscience, New York, 1963.

15 W. Kemula. Microchem. J. 11, 54 (1966).

W. Kemula and S. Sacha. Microchemical J. 11, 62 (1966).

$16 \mathrm{~W}$. Kemula and S. Sacha, In press

17 W. Kemula and J. W. Strojek. Chem. Anal. Warsaw 10, 1333 (1965).

18 W. Kemula and S. Głodowski. Roczniki Chem. 36, 1203 (1962), Chem. Anal. Warsaw 11, 403 (1966).

19 G. Semerano and L. Riccoboni. Gazz. chim. ital. 72, 297 (1942).

H. M. Davies and I. E. Seaborn. Advances in Polarography, Vol. 1, p. 239. Pergamon Press, Oxford, 1960.

20 W. Kemula and J. Strojek, In press. 